\title{
Análisis de los factores que determinan la ocupación de cajas-nido para aves insectívoras en parques urbanos de Donostia/San Sebastián.
}

\section{Factors shaping nest-box occupancy rate of insectivorous birds in urban parks in Donostia/San Sebastián.}

Maite Laso ${ }^{1 *}$, Urtzi Iturriotz ${ }^{1}$, Sergio Delgado ${ }^{1}$, Nere Zorrozua ${ }^{1,2}$, Nerea Pagaldai ${ }^{1}$, Beñat Díaz ${ }^{1}$, Juan Arizaga ${ }^{1}$

\section{Resumen}

Las cajas-nido constituyen una herramienta muy útil para el desarrollo de diversos estudios en aves, pues permiten el diseño experimental y la comparación de muestras sometidas a distintos tratamientos o localizaciones. Igualmente, son útiles para la conservación de aves en hábitats con muy poco arbolado maduro, como ocurre en muchos parques urbanos. Con el fin de impulsar la biodiversidad en el ámbito urbano, la Sociedad de Ciencias Aranzadi inició en 2018 el proyecto Hirilife, entre cuyas acciones estaba la colocación de cajasnido para aves paseriformes insectívoras en arbolado en calles, paseos y parques urbanos de la ciudad de San Sebastián. Este artículo se presenta con el fin de (1) determinar la tasa de ocupación de estas cajas-nido; y (2) explorar los factores que condicionan esta ocupación, con el fin de establecer, a futuro, una guía de buenas prácticas orientada a optimizar

1 Sociedad de Ciencias Aranzadi. Departamento de Ornitología.

Alto Zorroaga 11. 20014 Donostia-San Sebastian. Gipuzkoa.

\section{Departamento de Zoología y Biología Celular Animal.}

Universidad del País Vasco UPV/EHU, Sarriena s/n, 48940 Leioa.

*Correspondencia autor: mlaso@aranzadi.eus 
su colocación. A lo largo de la primavera de 2020 se revisaron 202 cajas en total, de las 220 que se habían colocado. Se ocuparon un total de 91 (45\%). La tasa de ocupación, no obstante, varió significativamente entre zonas. Las cajas en las que se pudo determinar la especie fueron ocupadas por dos especies de páridos: el herrerillo común Cyanistes caeruleus y el carbonero común Parus major. Se observa una dominancia del carbonero común (31 versus 10 cajas; el resto se corresponde con las que no se pudo determinar la especie). Según los modelos las cajas son ocupadas con más probabilidad cuando se sitúan en entornos con una gran cantidad de hábitat natural.

Palabras clave: Páridos, Cyanistes caeruleus, Parus major, arbolado maduro, biología de la conservación, ciudades verdes.

\begin{abstract}
Nest-boxes constitute a very useful tool in ornithological studies, since they allow the development of experimental designs and permit comparisons to be made between samples subjected to different treatments and locations. Moreover, they are also useful for bird conservation in habitats with low amounts of mature trees, as occurs in many urban parks. The 'Hirilife' project, developed by the Aranzadi Sciences Society, was launched in 2018 with the aim of promoting biodiversity in urban areas. This project included actions oriented to install and monitor nest-boxes for insectivorous passerine birds in the streets, promenades and parks of the city of San Sebastian. As a result, in this article we (1) describe the nest-box occupation rate, and (2) examine those factors that determine this rate, with the aim of drawing up a good practice guide to optimize the installation of new nestboxes in the future. During the spring of 2020, 202 nest-boxes were surveyed. Overall, 91 were occupied (45\%). The occupation rate, however, varied substantially between zones. The nest-boxes were occupied by two species: the blue tit Cyanistes caeruleus and the great tit Parus major. The latter species was dominant (31 versus 10 nest-boxes). According to the model of nest-box, these were more likely to be occupied when they were situated in areas close to/surrounded by a high amount of natural habitat.
\end{abstract}

Keywords: Paridae, Cyanistes caeruleus, Parus major, mature woodland, conservation biology, green urban areas.

\title{
Laburpena
}

Habia-kutxak hegaztien hainbat ikerketa egiteko oso tresna erabilgarriak dira; izan ere, diseinu esperimentala eta tratamendu ezberdinen laginen konparaketa egiteko aukera ematen dute. Era berean, zuhaitz heldu gutxi dauden habitatetan hegaztiak kontserbatzeko baliagarriak dira, hiriko parke askotan gertatzen den bezala. Hiri-eremuan biodibertsitatea areagotzeko helburuarekin, Aranzadi Zientzia Elkarteak 'Hirilife' proiektua martxan jarri zuen 2018an. Proiektu honetako ekintzen artean hegazti paseriforme intsektujaleen habia-kutxak kokatu ziren Donostiako kaleetako zuhaitzetan, pasealekuetan eta hiriko par- 
keetan. Artikulu honen helburua (1) habia-kutxen okupazio-tasa zehaztea da; eta (2) okupazio hori baldintzatzen duten faktoreak aztertzea da, etorkitzun hurbilean horien kokapena hobetzeko jardunbide egokiak ezartzeko. 2020ko udaberrian 220 habia-kutxetatik 202 aztertu ziren. Guztira, 91 (\%45) okupatu ziren. Hala ere, okupazio-tasa nabarmen aldatu zen eremuen artean. Espeziea zehaztea possible izan zen habia-kutxetan bi parido espezie zeuden: amilotx urdina Cyanistes caeruleus eta kaskabeltz handia Parus major. Kaskabeltz handia nagusi izan zen (31tik 10 habia-kutxa; gainerakoetan ez zen espeziea zehaztu). Ereduen arabera habia-kutxak okupatuak izateko probabilitatea handiagoa da ingurunean habitat natural gehiago dutenean.

Gako hitzak: Paridoak, Cyanistes caeruleus, Parus major, zuhaitz helduak, kontserbazio biologia, hiri berdeak.

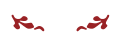

\section{Introducción}

La expansión urbana es un fenómeno asociado con cambios tanto bióticos como abióticos, que acarrean consecuencias sobre la fauna silvestre (Grimm et al., 2008). Los procesos biológicos y ecológicos que determinan la distribución y abundancia de las especies que son capaces de colonizar el ambiente urbano y de los factores que condicionan esta colonización son todavía hoy objeto de análisis (Chamberlain et al., 2009, Ryder et al., 2010, Murgui \& Hedblom, 2017, Ossola \& Niemelä, 2018, Saccavino et al., 2018, Méndez et al., 2020). Se sabe que, en cuanto a la avifauna, en general el proceso de urbanización reduce la riqueza de especies pero aumenta la densidad total de estas (Hohtola, 1978, Bezzel, 1985, Jokimäki \& Suhonen, 1993, McKinney, 2008). También cabe destacar, como es lógico, que en los parques urbanos la diversidad de especies es mayor que en otras áreas urbanas (Hadidian et al., 1997, Jokimäki, 1999, Anton et al., 2017, Ibáñez-Álamo et al., 2017, Oliveira Hagen et al., 2017).

La urbanización conlleva la desaparición de hábitat natural, incluidos los bosques con árboles maduros, cuyos agujeros son usados por multitud de especies para criar. En este contexto, uno de los grupos de aves más afectados por la urbanización son las que crían en las cavidades que se generan en árboles viejos. Así, la falta de estas cavidades puede ser un gran limitante en el establecimiento de poblaciones estables en el ámbito urbano, acentuándose la limitación de un recurso que ya de por sí es escaso en la naturaleza (Newton, 1994, Cockle et al., 2011, Wiebe, 2011, Reynolds et al., 2019). En este contexto, las cajas-nido ofrecen una solución viable para paliar este déficit de oquedades (e.g., Jokimäki, 1999). En ocasiones, no obstante, su eficiencia ha sido puesta en duda debido a las bajas tasas de ocupación o la ocupación de las cajas por especies no objetivo (Grarock et al., 2013, Williams et al., 2013). En términos 
globales, el uso de cajas-nido en medios urbanizados mejora la presencia de especies de aves autóctonas y contribuye a aumentar la diversidad (Jokimäki, 1999).

Por otro lado, las cajas-nido constituyen una herramienta muy útil para el desarrollo de diversos estudios en aves, pues permiten el diseño experimental y la comparación de muestras sometidas a distintos tratamientos o localizaciones (Oppliger et al., 1994, Sanz, 2002, Barba et al., 2009, Nicolaus et al., 2018, Samplonius et al., 2018). En un gradiente urbano-rural las cajas-nido pueden servir para explorar el efecto de la urbanización sobre las aves.

Con el fin de impulsar la biodiversidad en el ámbito urbano, la Sociedad de Ciencias Aranzadi inició en 2018 un proyecto, Hirilife (www.hirilife.eus), entre cuyas acciones estaba la colocación de cajas-nido para aves paseriformes insectívoras en arbolado en calles, paseos y parques urbanos de la ciudad de San Sebastián. Este artículo se presenta con el fin de determinar (1) la tasa de ocupación de estas cajas-nido; y (2) los factores que condicionan esta ocupación, con el fin de establecer, a futuro, una guía de buenas prácticas orientada a optimizar su colocación.

\section{Material y métodos}

\section{Área de estudios}

Este estudio se realizó en el término municipal de San Sebastián (Gipuzkoa). Con 181.652 habitantes, el municipio se extiende por una superficie de 6.100 ha (fuente: Eustat, 2019), en un rango altitudinal entre 0 y 585 m.s.n.m. La temperatura media anual se sitúa en $15^{\circ} \mathrm{C}$ y la precipitación, en unos $1500 \mathrm{~mm}$. Biogeográficamente, el municipio se sitúa en la región eurosiberiana, provincia cántabro atlántica, en el piso termotemplado, por lo que el hábitat potencial natural estaría formado, principalmente, por las series de vegetación robledal-fresneda mesofítica, robledales acidófilos, marojales y encinares cantábricos, así como alisedas en cursos fluviales (Loidi et al., 2011). En la actualidad, S. Sebastián está constituido por una trama urbana densa que sobre todo hacia el oeste, sur y, parcialmente, el este (sureste), se transforma en un mosaico agro-forestal típico de paisaje de campiña. La ciudad, no obstante, cuenta con un número destacable de espacios verdes y grandes parques urbanos (Fig. 1).

\section{Protocolo de colocación y revisión de cajas}

Se colocaron un total de 220 cajas-nido, repartidas en 14 zonas, fundamentalmente parques urbanos (para más detalles ver Anexo 1; Fig. 1). Fueron puestas durante el periodo no reproductor de 2018 y, fundamentalmente, 2019 (hasta enero de 2020). En el caso de las cajas que se habían colocado en 2018 , se procedió a su revisión y limpieza con el fin de dejarlas vacía y utilizables para la temporada de cría de 2020. 


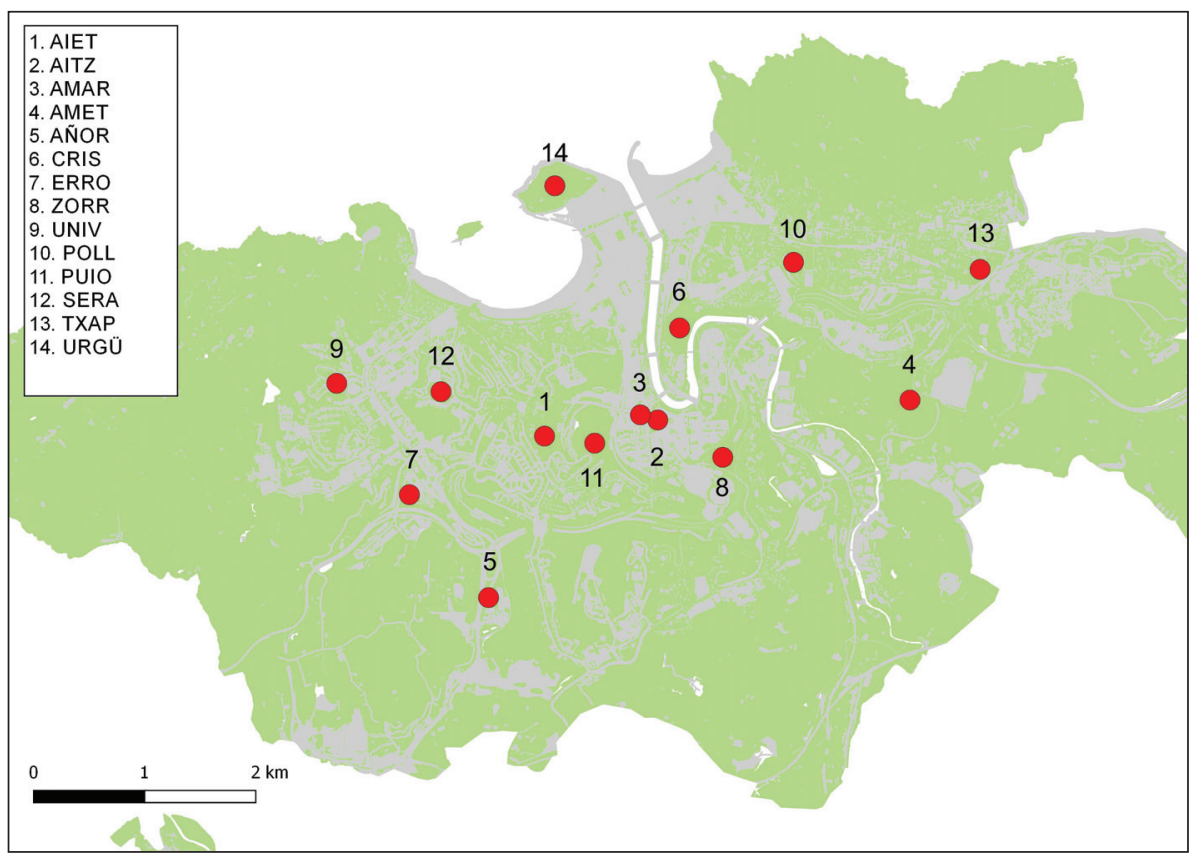

Fig. 1.- Distribución espacial de las zonas del municipio de Donostia/San Sebastián donde se colocaron cajas-nido.

Fig. 1.- Spatial distribution of the zones where the nest-boxes were installed in the city of Donostia/San Sebastián.

Se utilizaron 3 tipos de cajas: (1) modelo Diputación (150; diámetro del orificio de entrada: $35 \mathrm{~mm}$ ), (2) modelo Ayuntamiento (55, de la casa The Green Tit Company; diámetro: $30 \mathrm{~mm}$ ), (3) modelo Oryx (15; diámetro, $26 \mathrm{~mm}$ ).

Cada caja se colocó en una rama que, en lo posible, no tuviera cerca otras ramas o troncos, con el fin de evitar el acceso de posibles depredadores. Además, todas las cajas fueron numeradas y geolocalizadas. Durante la colocación se tuvieron en cuenta los siguientes principios: (1) ubicarlas a una altura de 3 a 4 m, para reducir en lo posible la depredación así como las molestias de origen humano (Kali ski et al., 2014, Maziarz et al., 2016), pero a la vez facilitar que pudieran ser revisadas con posterioridad; (2) aplicar densidad general de 10-20 cajas/ha y distancia mínima entre ellas de $5 \mathrm{~m}$.

La revisión durante el periodo de cría de 2020 abarcó del 15 de abril hasta el 15 de junio, procurando llevar a cabo una revisión cada 15 días. El objetivo de la misma fue saber si la caja había sido ocupada y determinar, cuando se pudo, el tamaño de puesta, el número de pollos y la especie que ocupaba la caja (en este artículo no se han analizado los parámetros reproductivos). Fueron consideradas como ocupadas las cajas 
que tuvieron nido, tanto si luego éste se abandonó como si no (esto es, sí llegó a haber puesta). No fueron consideradas como ocupadas las cajas que se hallaron vacías, ocupadas por especies que no fueran aves (roedores, insectos, etc.) o en las que se registraron nidos a medio construir, con muy poco aporte de material. La revisión de cada caja se desarrolló lo más rápido posible y se evitaron los días de fuerte viento y/o Iluvia para minimizar molestias. Para ver el contenido de las cajas se empleó una pértiga o una cámara endoscópica. Debido al COVID-19 y al Estado de Alarma, la revisión de las cajas fue realizada por profesionales con los correspondientes permisos administrativos.

Asimismo, en cada una de las cajas se llevó a cabo una caracterización del entorno, con el fin de, posteriormente, tener en cuenta esas variables para el análisis de los factores que determinan la probabilidad de ocupación:

(1) Porcentaje (cobertura) de hábitats, para un radio de $250 \mathrm{~m}$ : agua (cuerpos de agua, como balsas o estanques, sin incluir los ríos), ríos, forestal, matorral, pastos y prados, cultivos, jardines, edificio, urbanizado no edificado (e.g., carreteras, plazas, aceras...).

(2) Características de la caja: orientación, altura, anchura del árbol (a ca. 1,5 m sobre el suelo). El tipo de modelo no se incluyó en el estudio porque no hubo un diseño equitativo de colocación de diferentes tipos de cajas en cada uno de los parques.

(3) Características del entorno más cercano a la caja (radio: 25 m): número de árboles y anchura media de éstos, distancia a camino y tipo de camino (camino pavimentado/no pavimentado, calle), porcentaje de superficie cubierta por arbolado y tipo de arbolado (caducifolio, perennifolio), porcentaje de superficie cubierta por zarzas, arbustos, vegetación herbácea, edificios, pavimento u otros tipos de ocupación.

\section{Análisis estadísticos}

Para determinar, primeramente, si la tasa de ocupación varió entre zonas, se aplicó un test de chi-cuadrado.

A continuación, se llevaron a cabo análisis con el fin de determinar qué factores son los que influyeron en la probabilidad de que una caja se ocupara o no. Sólo se consideraron para el análisis aquellas cajas en las que se había medido tanto la ocupación (si/no) como el total de 28 variables predictoras arriba descritas. Puesto que el número de variables predictoras era muy alto, se llevaron a cabo técnicas de reducción de variables (Análisis de Componentes Principales, ACP) y una modelización por pasos, hasta llegar al modelo definitivo. A continuación se describe todo este proceso en detalle: 
(1) Primeramente, se aplicó un ACP $\left(\mathrm{ACP}_{\text {macro }}\right)$ para el porcentaje de hábitats en radio

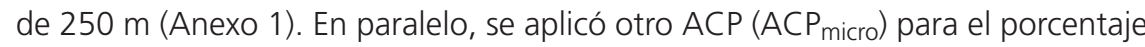
de coberturas en radio de 25 m (Anexo 1). Para cada uno de los ACP, los Componentes con autovalores $>1$ se incluyeron en los modelos que se utilizaron para determinar los factores que influyeron en la probabilidad de ocupación.

(2) Tras los ACP, se llevaron a cabo tres modelizaciones a través de un Modelo Mixto Lineal Generalizado (MMLG) en cada caso, para determinar cuál tenía un mejor ajuste. Concretamente, teniendo en todos los casos la ocupación de la caja (si/no, codificada como 1/0; variable ocup en la ecuación) como variable objeto, se hizo un MMLG considerando los Componentes del $A C P_{\text {macro }}(\mathrm{m} 1)$, o bien las variables que se relacionaron con las características de la caja (m2), o los Componentes del

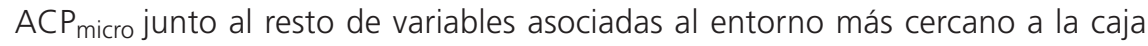
como variables/factores independientes (m3) (notación según programa R):

$m 1=$ ocup $\sim C P 1_{\text {macro }}+C P 2_{\text {macro }}+C P 3_{\text {macro }}$

$m 2$ = ocup $\sim$ orient + altu.caja + anch.sust (donde: orient es la orientación de la caja, altu.caja es la altura de la caja y anch.sust es el ancho del árbol donde se colocó la caja).

m3 $=$ ocup $\sim$ pies + anch.pies + dist.cami*tipo.cami $+C P 1_{\text {micro }}+C P 2_{\text {micro }}+C P 3_{\text {micro }}$ + CP4 micro (donde: pies es el número de árboles del entorno, anch.pies es la anchura media de esos árboles, dist.cami y tipo.cami es la distancia al camino más próximo así como el tipo de camino; la interacción se debe a que el efecto de la distancia pudo depender del tipo de camino -no sería lo mismo un camino para peatones que una calle con tráfico-).

En todos estos modelos, además, se añadió el parque como factor aleatorio, con el fin de controlar el hecho de que la ocupación dentro de cada parque no es totalmente independiente. En todos los casos se utilizó una función de enlace logit con una distribución binomial de errores. Posteriormente, se calculó el valor Akaike (AIC) de cada modelo para determinar cuál tenía un mejor ajuste a datos (Akaike, 2011). Diferencias de AIC inferiores a 2 fueron consideradas como evidencia de que un modelo no difería respecto a otro (Burnham \& Anderson, 1998). El AIC más bajo fue obtenido por el modelo $m 1(252,10 ; m 2=264,30 ; m 3=260,32)$. En consecuencia, solamente $m 1$ tuvo un ajuste a los datos mejor que los otros dos modelos. La sobredispersión de este modelo $m 1$ es 0,966 (por lo que el ajuste a los datos fue bueno) y el valor de $R^{2}$, 0,20 (esto es, un 20\% de la varianza fue explicada por el modelo). Sobre el modelo saturado que incluyó las variables del modelo $m 1$ se aplicó la función 'dredge' del paquete 'MuMIn' (Barton, 2014) con el fin de ejecutar un proceso de selección de variables del que se obtuviera, finalmente, un ranking de modelos, basado en valores AIC, para determinar la lista de la(s) variables que contribuyeron con más peso a explicar la varianza de nuestros datos. 


\section{Resultados}

A lo largo de la primavera de 2020 se revisaron 202 cajas en total, de las 220 que se habían colocado. La diferencia se debe principalmente a la desaparición de cajas y causas logísticas. Se ocuparon un total de 91 (45\%). La tasa de ocupación, no obstante, varió significativamente entre zonas $\left(\chi^{2}=37,14 ; P<0,001\right)$, desde un valor de 0\% (AMAR, AITZ) hasta un valor de 90\% (UNIV; para más detalles ver Tabla 1).

\begin{tabular}{|l|c|c|c|}
\hline \multirow{2}{*}{ Zona (código) } & \multirow{2}{*}{ Superficie(ha) } & \multicolumn{2}{|c|}{ Ocupación } \\
\cline { 2 - 4 } & & No & Sí \\
\hline AIET & 15 & 7 & $13(65 \%)$ \\
\hline AITZ & $<2$ & 10 & $0(0 \%)$ \\
\hline AMAR & $<2$ & 5 & $0(0 \%)$ \\
\hline AMET & 50 & 13 & $16(55 \%)$ \\
\hline AÑOR & $<2$ & 4 & $6(60 \%)$ \\
\hline CRIS & 10 & 10 & $10(50 \%)$ \\
\hline ERRO & $<2$ & 8 & $2(60 \%)$ \\
\hline ZORR & 4 & 6 & $9(25 \%)$ \\
\hline UNIV & 2 & 1 & $2(22 \%)$ \\
\hline POLL & $<2$ & 7 & $6(60 \%)$ \\
\hline PUIO & 15 & 4 & $5(33 \%)$ \\
\hline SERA & 3 & 10 & $4(25 \%)$ \\
\hline TXAP & $<2$ & 12 & $6(30 \%)$ \\
\hline URGÜ & 20 & 14 & $20 \%)$ \\
\hline
\end{tabular}

Tabla 1.- Número de cajas-nido colocadas en cada zona y su ocupación. Todas las zonas son parques urbanos.

Table 1.- Number of nest-boxes placed in each zone and its occupation rate. All the zones were urban parklands.

El $100 \%$ de las cajas que se pudo determinar la especie fueron ocupadas por dos especies de páridos: el herrerillo común Cyanistes caeruleus y el carbonero común Parus major. Se observa una dominancia del carbonero común (31 versus 10 cajas; en el resto no se pudo determinar la especie).

En el Anexo 2 se resume el valor medio de los parámetros que describen el hábitat del entorno de las cajas así como su ubicación en el árbol. En promedio, puede verse, entre otras variables, que las cajas se situaron en zonas muy urbanizadas con un alto porcentaje de superficie ajardinada, a algo más de $3 \mathrm{~m}$ de altura en un árbol de 0,5 $\mathrm{m}$ de diámetro, rodeado de un elevado número de árboles de similar grosor y a 8,5 $\mathrm{m}$ de distancia de un camino. 
Como ya se ha adelantado parcialmente en Métodos, el modelo $m 3$, relativo al efecto de las características del entorno más cercano (radio $25 \mathrm{~m}$ ) a la caja, o el modelo $\mathrm{m} 2$, relativo a las características del árbol donde se colocó la caja, no tuvieron un ajuste a los datos tan bueno como el modelo $\mathrm{m} 1$, que tuvo en cuenta el hábitat a una escala más amplia (radio 250 m). Tras el proceso de selección de variables, tuvimos tres modelos con una diferencia de AIC $<2$ unidades. En su conjunto, las variables que se incluyeron fueron el CP1 macro a CP3 macro. Al correr, en consecuencia, el modelo saturado $m 1$ se vio que solo el $C P 1_{\text {macro }}$ afectó significativamente a la probabilidad de ocupación. Observamos, concretamente, que ésta se incrementa al aumentar el valor de PC $1_{\text {macro, }}$ esto es, las cajas son ocupadas con más probabilidad cuando se sitúan en entornos con una gran cantidad de hábitat natural (Fig. 2).

\section{Discusión}

Este artículo aborda, por primera vez para San Sebastián, el análisis de los factores que determinan la probabilidad de ocupación de cajas-nido para aves. Es, además, uno de los pocos que tratan esta temática en el conjunto de la franja Cantábrica (De la Hera et al., 2013). Aunque descriptivo, se aporta de este modo información útil para el diseño y gestión de proyectos de implementación y seguimiento de cajas-nido

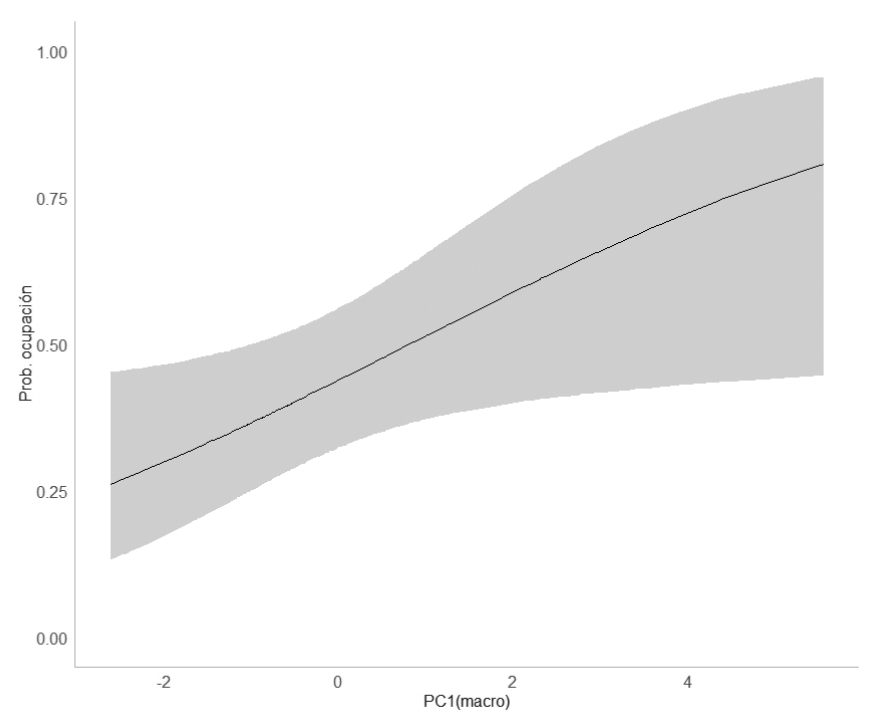

Fig. 2.- Probabilidad de ocupación ( $1=100 \%$; \pm intervalo de confianza al 95\%, área en gris) de cajasnido según el valor de PC 1 macro.

Fig. 2.- Nest-boxes occupation probability ( $1=100 \% ; \pm 95 \%$ confidence interval, grey area) in relation to $P C 1$ macro values. 
para aves insectívoras (páridos) en medios urbanizados. Se contribuye, así, a incrementar el conocimiento básico en materia de biodiversidad urbana (Ossola \& Niemelä, 2018), aplicada a una ciudad del área cantábrica.

En términos globales, la tasa de ocupación de las cajas fue alta (43,08\%). Debe considerarse, además, que son cajas colocadas justo unos meses antes de su revisión en 2020 (la mayor parte se colocó en otoño de 2019) y que la tasa de ocupación a menudo se incrementa con los años (Rey et al., 2017). Una tasa de ocupación tan alta como la observada en S. Sebastián indica, en término globales, déficit de cavidades naturales (Lindenmayer et al., 2009, Berthier et al., 2012) que, por lo tanto, podría ser uno de los factores limitantes para el crecimiento de las poblaciones que se reproducen en todos estos parques. De ello se deriva la importancia de promover la colocación y, posteriormente, conservación y mantenimiento de cajas-nido en los parques urbanos del municipio. En paralelo, también se debería garantizar la presencia de árboles maduros, que son los que posibilitan la existencia de huecos, no ya sólo para pequeñas aves insectívoras, sino también para otras aves (e.g., cárabos) así como otros grupos faunísticos (e.g., mustélidos y lirones). En este contexto, la realización de podas puede, también, potenciar la generación de huecos (Avilés, 2019). En todo caso, mientras siga existiendo este déficit de huecos, se pone de manifiesto que las cajas-nido son útiles. Gracias a éstas, así, se contribuye a la presencia de poblaciones estables de aves insectívoras, las cuales pueden jugar un papel fundamental en el control de plagas, como la de la procesionaria u otras (Ceballos, 1968, Solomon et al., 1976, Sanz, 2001).

Sólo fueron dos las especies que ocuparon las cajas (a falta de determinar la especie en algunas de las cajas ocupadas): el carbonero común y el herrerillo común. Aproximadamente tres de cada cuatro cajas fueron ocupadas por carboneros. Esto podría ser debido al tamaño del agujero de entrada. Este tamaño, relativamente grande, favorece a las especies más grandes, como el carbonero, frente a las más pequeñas, como el herrerillo (Lambrechts et al., 2010). Así, el orificio óptimo para el carbonero es de 32 mm, mientras que para el herrerillo es 26 mm (Lambrechts et al., 2010). Por otro lado, no debe obviarse que el carbonero es una especie más abundante que el herrerillo. Así, según el atlas de aves nidificantes de S. Sebastián, actualmente en desarrollo, la población de carboneros comunes se calcula en unos 3.000 individuos (adultos), mientras que la de los herrerillos se estima en unos 1.500 individuos (Sociedad de Ciencias Aranzadi, en prep.). Este ratio poblacional entre ambas especies es similar al hallado en otros estudios (Solonen, 2001).

La variable que más influyó en la probabilidad de que una caja se ocupara fue el primer componente de un ACP (PC1 macro). Este componente se asoció positivamente a la superficie de hábitat natural, sobre todo masas forestales y prados/pastizales (Anexo 1), esto es, paisaje de campiña. La disponibilidad de huecos es habitualmente baja, por lo que la competencia por este recurso es en general alta (Newton, 1994, Wiebe, 2011). El número de huecos se incrementa en hábitats forestales maduros donde 
existen árboles viejos (Maziarz et al., 2016). En consecuencia, las poblaciones de las especies que dependen de este tipo de huecos para criar suelen ser también mayores en aquellos hábitats en los que la abundancia de arbolado maduro es mayor, como pueden ser las masas forestales de hábitat natural frente al arbolado típico de ciertos parques y jardines (Jokimäki, 1999). Probablemente, el efecto positivo del PC 1 macro sobre las tasas de ocupación se deba a una probabilidad más alta de colonización en aquellos entornos/hábitats donde, posiblemente, el número de aves fuera también mayor. Además de la densidad de aves, otros factores tales como la heterogeneidad del paisaje urbano podrían, también, tener un papel destacado a la hora de explicar nuestros resultados (Szulkin et al., 2020). Así, cierto tipo de ambientes urbanos podrían favorecer la presencia de individuos de 'baja' calidad, en comparación con aquellos que, teóricamente, ocuparían los hábitats más adecuados tales como masas forestales (Björklund et al., 2009) (Tabla 2).

\begin{tabular}{|lcc|}
\hline Efecto fijo & Beta parámetros $( \pm$ SE) & $P$ \\
\hline$P C 1_{\text {macro }}$ & $+0,30 \pm 0,14$ & 0,032 \\
\hline$P C 2_{\text {macro }}$ & $-0,05 \pm 0,16$ & 0,735 \\
\hline$P C 3_{\text {macro }}$ & $+0,22 \pm 0,22$ & 0,302 \\
\hline
\end{tabular}

Tabla 2.- Resultados un modelo mixto lineal generalizado que analizó los efectos de PC1 macro a PC3macro sobre la probabilidad de ocupación.

Table 2.- Results of a generalized linear mixed model in which the PC 1 macro to PC 3 macro effect on occupation rate was examined.

\section{Agradecimientos}

Participaron en la revisión y colocación de cajas: J. M. Aranjuelo, C. Bengoa, G. Bregaña, M. Elena, J. M. Elkoro-Uribe, T. Enríquez, M. L. García, Á. García, I. Ibero, I. Intxaurrandieta, A. Iñurritegi, D. Jacome, I. Larrañaga, A. Lasa, I. Mendizabal, J. Querejeta, G. Rández, P. Rodríguez, Y. Sagarminaga, P. Sancho, C. Ugarte, M. Varona, J. M. Yetano. El Ayuntamiento de San Sebastián autorizó la colocación de las cajas y financió una parte del trabajo. La Diputación de Gipuzkoa proporcionó varias de las cajas que fueron utilizadas en este proyecto. P. Salmón y un revisor anónimo aportaron valiosos comentarios que contribuyeron a mejorar una primera versión del trabajo.

\section{Bibliografía}

Akaike, H., 2011. Akaike's Information Criterion. In: M. Lovric (Eds.), International Encyclopedia of Statistical Science, 25-25. Springer Berlin Heidelberg, Berlin, Heidelberg.

Anton, M., Herrando, S., Garcia, D., Ferrer, X., Cebrian, R., 2017. Atlas dels ocells nidificants de Barcelona. Ayuntament de Barcelona/ICO/UB/Zoo, Barcelona. 
Avilés, J.M., 2019. Pruning promotes the formation of an insufficient number of cavities for hollow-dependent birds in Iberian Holm-oak dehesas. Forest Ecology and Management 453,117627

Barba, E., Atiénzar, F., Marín, M., Monrós, J.S., Gil Delgado, J.A., 2009. Patterns of nestling provisioning by a single prey loader bird, Great Tit Parus major. Bird Study 56, 187-197.

Barton, K., 2014. MuMIn: Multi-model inference. R package version 1.10.5. Vienna, Austria.

Berthier, K., Leippert, F., Fumagalli, L., Arlettaz, R,. 2012. Massive nest-box supplementation boosts fecundity, survival and even immigration without altering mating and reproductive behaviour in a rapidly recovered bird population. Plos One 7, e36028.

Bezzel, E., 1985. Birdlife in intensively used rural and urban environments. Ornis Fennica 62, 90-95.

Björklund, M., Ruiz, I., Senar, J.C., 2009. Genetic differentiation in the urban habitat: the great tits (Parus major) of the parks of Barcelona city. Biological Journal of the Linnean Society 99, 9-19.

Burnham, K. P., Anderson, D.R., 1998. Model Selection and Inference. A Practical Information Theoretic Approach. Springer-Verlag, New York.

Ceballos, P., 1968. Protección de aves insectívoras. Boletín del Servicio de Plagas Forestales 26, 62-70.

Cockle, K.L., Martin, K., Wesolowski, T., 2011. Woodpeckers, decay, and the future of cavity-nesting vertebrate communities worldwide. Frontiers in Ecology and the Environment 9, 377-382.

Chamberlain, D.E., Cannon, A.R., Toms, M.P., Leech, D.I., Hatchwell, B.J., Gaston, K.J., 2009. Avian productivity in urban landscapes: a review and meta-analysis. Ibis 151(1), 1-18.

De la Hera, I., Arizaga, J., Galarza, A., 2013. Exotic tree plantations and avian conservation in northern Iberia: a view from a nest-box monitoring study. Animal Biodiversity and Conservation 36, 153-163.

Grarock, K., Lindenmayer, D.B., Wood, J.T., Tidemann, C.R., 2013. Does human-induced habitat modification influence the impact of introduced species? A case study on cavitynesting by the introduced common myna (Acridotheres tristis) and two Australian native parrots. Environ Manage 52, 958-970.

Grimm, N.B., Faeth, S.H., Golubiewski, N.E., Redman, C.L., Wu, J., Bai, X., Briggs, J.M., 2008. Global change and the ecology of cities. Science 319, 756.

Hadidian, J., Sauer, J., Swarth, C., Handly, P., Droege, S., Williams, C., Huff, J., Didden, G., 1997. A citywide breeding bird survey for Washington, D.C. Urban Ecosystems 1, 87-102.

Hohtola, E., 1978. Differential changes in bird community structure with urbanisation: a study in Central Finland. Ornis Scandinavica 9, 94-100.

Ibáñez-Álamo, J.D., Rubio, E., Benedetti, Y., Morelli, F., 2017. Global loss of avian evolutionary uniqueness in urban areas. Global Change Biology 23, 2990-2998. 
Jokimäki, J., 1999. Occurrence of breeding bird species in urban parks: Effects of park structure and broad-scale variables. Urban Ecosystems 3, 21-34.

Jokimäki, J., Suhonen, J., 1993. Effects of urbanization on the breeding bird species richness in Finland: A biogeographical comparison. Ornis Fennica 70, 71-77.

Kali ski, A., Wawrzyniak, J., Ba bura, M., Skwarska, J., Zieli ski, P., Gl dalski, M., Ba bura, J., 2014. Does the threat of European Pine Marten (Martes martes) predation influence the height of nests built by Blue Tits (Cyanistes caeruleus) and Great Tits (Parus major)? Avian Biology Research 7, 83-90.

Lambrechts, M.M., Adriaensen, F., Ardia, D.R., Artemyev, A.V., Atiénzar, F., Banbura, J., Barba, E., Bouvier, J.-C., Camprodon, J., Cooper, C.B., 2010. The design of artificial nestboxes for the study of secondary hole-nesting birds: a review of methodological inconsistencies and potential biases. Acta Ornithologica 45, 1-26.

Lindenmayer, D.B., Welsh, A., Donnelly, C., Crane, M., Michael, D., Macgregor, C., McBurney, L., Montague-Drake, R., Gibbons, P., 2009. Are nest boxes a viable alternative source of cavities for hollow-dependent animals? Long-term monitoring of nest box occupancy, pest use and attrition. Biological Conservation 142, 33-42.

Loidi, J., Biurrun, I., Campos, J.A., García-Mijangos, I., Herrera, M., 2011. La vegetación de la Comunidad Autónoma del País Vasco. Leyenda del mapa de series de vegetación a escala 1:50.000. Gobierno Vasco, Vitoria-Gastez.

Maziarz, M., Wesołowski, T., Hebda, G., Cholewa, M., Broughton, R.K., 2016. Breeding success of the Great Tit Parus major in relation to attributes of natural nest cavities in a primeval forest. Journal of Ornithology 157, 343-354.

McKinney, M.L., 2008. Effects of urbanization on species richness: A review of plants and animals. Urban Ecosystems 11, 161-176.

Méndez, A., Montalvo, T., Aymí, R., Carmona, M., Figuerola, J., Navarro, J., 2020. Adapting to urban ecosystems: unravelling the foraging ecology of an opportunistic predator living in cities. Urban Ecosystems 23, 1117-1126.

Murgui, E., Hedblom, M., 2017. Ecology and conservation of birds in urban environments. Springer, New York.

Newton, I., 1994. The role of nest sites in limiting the numbers of hole-nesting birds: A review. Biological Conservation 70, 265-276.

Nicolaus, M., Barrault, S.C.Y., Both, C., 2018. Diet and provisioning rate differ predictably between dispersing and philopatric pied flycatchers. Behavioral Ecology 30, 114-124.

Oliveira Hagen, E., Hagen, O., Ibáñez-Álamo, J. D., Petchey, O. L., Evans, K.L., 2017. Impacts of urban areas and their characteristics on avian functional diversity. Front. Ecol. Evol. 5, 84.

Oppliger, A., Richner, H., Christe, P., 1994. Effect of an ectoparasite on lay date, nest-site choice, desertion, and hatching success in the great tit (Parus major). Behavioral Ecology 5, 130-134. 
Ossola, A., Niemelä, J., 2018. Urban biodiversity: from research to practice. Routledge (Taylor \& Francis Group), Oxon.

Rey, J.M., Meltzer, J., de las Heras-Bravo, D., Cayuela, L., 2017. Potential of pest regulation by insectivorous birds in Mediterranean woody crops. Plos One 12, e0180702.

Reynolds, J.S., Ibáñez-Álamo, J.D., Sumasgutner, P., Mainwaring, M.C., 2019. Urbanisation and nest building in birds: a review of threats and opportunities. Journal of Ornithology $160,841-860$.

Ryder, T.B., Reitsma, R., Evans, B., Marra, P., 2010. Quantifying avian nest survival along an urbanization gradient using citizen- and scientist-generated data. Ecological Applications 20, 419-426.

Saccavino, E., Krämer, J., Klaus, S., Tietze, D.T., 2018. Does urbanization affect wing pointedness in the Blackbird Turdus merula? Journal of Ornithology 159, 1043-1051.

Samplonius, J.M., Bartošová, L., Burgess, M.D., Bushuev, A.V., Eeva, T., Ivankina, E.V., Kerimov, A.B., Krams, I., Laaksonen, T., Mägi, M., Mänd, R., Potti, J., Török, J., Trnka, M., Visser, M.E., Zang, H., Both, C., 2018. Phenological sensitivity to climate change is higher in resident than in migrant bird populations among European cavity breeders. Global Change Biology 24, 3780-3790.

Sanz, J.J. 2001. Experimentally increased insectivorous bird density results in a reduction of caterpillar density and leaf damage to Pyrenean oak. Ecological Research 16, 387-394.

Sanz, J.J., 2002. Climate change and breeding parameters of great and blue tits throughout the western Palaearctic. Global Change Biology 8, 409-422.

Solomon, M.E., Glen, D.M., Kendall, D.A., Milsom, N.F., 1976. Predation of overwintering larvae of Codling Moth (Cydia pomonella (L.) by birds. Journal of Applied Ecology 13, 341 352.

Solonen, T., 2001. Breeding ofthe Great Tit and Blue Tit in urban and rural habitats in southern Finland. Ornis Fennica 78, 49-60.

Szulkin, M., Garroway, C.J., Corsini, M., Kotarba, A.Z., Dominoni, D., 2020. How to quantify urbanisation when testing for urban evolution? In: Szulkin, M., Munshi-South, J., Charmantier, A. (Eds.). Urban Evolutionary Biology, Oxford University Press, Oxford.

Wiebe, K.L., 2011. Nest sites as limiting resources for cavity-nesting birds in mature forest ecosystems: a review of the evidence. Journal of Field Ornithology 82, 239-248.

Williams, D.R., Pople, R.G., Showler, D.A., Dicks, L.V., Child, M.F., Zu Ermgassen, E.K.H. J., Sutherland, W.J., 2013. Bird Conservation: Global evidence for the effects of intervention. Pelagic Publishing, Exter.

Fecha de recepción/ Date of reception: 09/12/2020

Fecha de aceptación / Date of acceptance: 29/06/2021

Editor Asociado / Associate editor: Iván de la Hera 
Anexo 1. Resultados de los PCA llevados a cabo para resumir el hábitat en torno a las cajas en un radio de $250 \mathrm{~m}$ (PCA macro) y el porcentaje de superficie cubierta por distintas variables en un radio de 25 m (PCAmicro). Abreviaciones (1) para el PCAmacro: agua, superficie relativa de agua, agua; edif, edificios; agro, cultivos; fore, masa forestal; jard, zona ajardinada; mato, matorral; prad, prados/pastizales; ríos, cursos fluviales; urba, urbanizado (excluidos los edificios); (2) para el PCAmicro: cobert, superficie relativa de cobert, arbolado ('sombra' de la copa); caduci, arbolado de hoja caduca; perenn.anch, arbolado perennifolio no conífera; perenn.otro, conífera; zarzas, zarzal; arbust, arbustos, matorral; herbac, herbáceo; pavime, pavimento no edificado; edific, edificios; otro, otros tipos de cobertura.

Annex 1. Results from a PCA conducted to summarise the habitat around nest-boxes within a radius of $250 \mathrm{~m}$ (PCA $\left.A_{\text {macro }}\right)$ and the percentage of surface covered by different variables within a radius of $25 \mathrm{~m}$ (PCA micro $)$. Abbreviations (1) for the PCA macro: agua, relative surface covered by water; edif, buildings; agro, crops; fore, forest; jard, garden; mato, shrub; prad, meadow/pastures; ríos, rivers; urba, urban (excluding buildings); (2) for the PCAmicro: cobert, relative surface covered by trees caduci, deciduous; perenn.anch, non-conifer perennial; perenn.otro, conifer; zarzas, brumbles; arbust, shrubs; herbac, herbaceous; pavime, pavements (excluding buildings); edific, buildings; otro, others

\begin{tabular}{|lrrrr|}
\hline Variable: & PC1macro & PC2macro & PC3macro & PC4macro \\
agua & $+0,06$ & $-0,29$ & $-0,66$ & $-0,21$ \\
edif & $-0,43$ & $+0,37$ & $+0,01$ & $+0,13$ \\
agro & $+0,23$ & $+0,39$ & $-0,02$ & $-0,56$ \\
fore & $+0,48$ & $+0,11$ & $+0,25$ & $+0,35$ \\
jard & $-0,05$ & $-0,58$ & $+0,10$ & $+0,13$ \\
mato & $+0,34$ & $+0,18$ & $-0,41$ & $-0,18$ \\
prad & $+0,43$ & $+0,19$ & $+0,31$ & $-0,00$ \\
rios & $-0,16$ & $-0,25$ & $+0,48$ & $-0,68$ \\
urba & $-0,45$ & $+0,39$ & $-0,04$ & $+0,02$ \\
Autovalor & 3,05 & 2,08 & 1,26 & 0,82 \\
Varianza & 33,84 & 23,15 & 14,01 & 9,18 \\
\hline Variable: & $\mathrm{PC} 1$ micro & $\mathrm{PC} 2$ micro & $\mathrm{PC} 3$ micro & $\mathrm{PC} 4 \mathrm{micr}$ \\
cobert & $-0,36$ & $-0,13$ & $+0,40$ & $-0,13$ \\
caduci & $-0,47$ & $-0,14$ & $-0,39$ & $+0,11$ \\
perenn.anch & $+0,21$ & $+0,30$ & $+0,36$ & $+0,34$ \\
perenn.otro & $+0,44$ & $-0,01$ & $+0,25$ & $-0,33$ \\
zarzas & $-0,03$ & $-0,70$ & $+0,10$ & $-0,02$ \\
arbust & $+0,10$ & $-0,17$ & $+0,50$ & $+0,28$ \\
herbac & $-0,38$ & $+0,56$ & $+0,10$ & $-0,07$ \\
pavime & $+0,38$ & $-0,01$ & $-0,37$ & $-0,37$ \\
edific & $+0,30$ & $+0,18$ & $-0,20$ & $+0,28$ \\
otro & $+0,13$ & $-0,11$ & $-0,24$ & $+0,69$ \\
Autovalor & 2,97 & 1,59 & 1,52 & 10,51 \\
Varianza & 29,71 & 15,87 & 15,20 & \\
\hline
\end{tabular}


Anexo 2. Valores medios y rangos de las variables que describen el hábitat del entorno y las características del árbol donde fueron colocadas las cajas-nido. Abreviación de variables, como en el Anexo 1 y Métodos. Unidad de la variable, en paréntesis.

Annex 2. Mean and range values for those variables which describe the habitat around and tree characteristics where next-boxes were put. Variable abbreviations, as in Annex 1. Variable unit, in brackets.

\begin{tabular}{|lcc|}
\hline Variable & Promedio ( \pm desv. estándar) & Rango \\
\hline Radio $250 \mathrm{~m}$ & $2,5 \pm 8,0$ & $0-42,7$ \\
agua (\%) & $10,7 \pm 5,7$ & $0-24,0$ \\
edif (\%) & $1,2 \pm 2,3$ & $0-15,3$ \\
agro (\%) & $8,5 \pm 12,7$ & $0-45,2$ \\
fore (\%) & $35,7 \pm 12,1$ & $15,4-61,9$ \\
jard (\%) & $3,4 \pm 3,9$ & $0-19,4$ \\
mato (\%) & $1,0 \pm 2,5$ & $0-13,5$ \\
prad (\%) & $1,8 \pm 4,8$ & $0-22,5$ \\
rios (\%) & $35,3 \pm 12,7$ & $11,9-62,3$ \\
urba (\%) & & $10-95$ \\
\hline Radio 25 m & $56,3 \pm 20,7$ & $0-100$ \\
cobert (\%) & $83,4 \pm 23,0$ & $0-80$ \\
caduci (\%) & $6,3 \pm 11,4$ & $0-85$ \\
perenn.anch (\%) & $10,3 \pm 19,5$ & $0-80$ \\
perenn.otro (\%) & $10,0 \pm 16,6$ & $0-50$ \\
zarzas (\%) & $7,4 \pm 8,7$ & $0-100$ \\
arbust (\%) & $65,2 \pm 22,9$ & $0-95$ \\
herbac (\%) & $13,7 \pm 15,5$ & $0-40$ \\
pavime (\%) & $2,0 \pm 5,6$ & $0-40$ \\
edific (\%) & $1,7 \pm 5,6$ & $5-110$ \\
otro (\%) & $49,8 \pm 21,1$ & $15-60$ \\
pies & $41,5 \pm 17,4$ & \\
anch.pies (cm) & $3,3 \pm 0,4$ & \\
\hline Árbol de la caja & $51,2 \pm 25,8$ & \\
altu.caja (m) & $8,5 \pm 6,5$ & \\
anch.sust (cm) & & \\
dist.cami (m) & & \\
\hline & & \\
\hline
\end{tabular}

